\title{
The Use of Carotid Artery Ultrasonography in Different Clinical Conditions
}

\author{
Armen Yuri Gasparyan ${ }^{1,2, *}$
}

\author{
${ }^{I}$ Department of Internal Medicine, Krankenhaus der Barmherzigen Brüder, Teaching Hospital of Medical University of \\ Graz, Graz, Austria \\ ${ }^{2}$ Dudley Group of Hospitals NHS Trust, West Midlands, UK
}

\begin{abstract}
B-mode ultrasonography of the carotid artery is a non-invasive, informative and reproducible technique used for the assessment of prevalence and course of atherosclerosis in a variety of clinical conditions. Visualization of intima-media complex, atherosclerotic plaques, rough arterial wall and calcifications of the carotid artery may be useful for the assessment of atherosclerotic burden. The latter was confirmed in a recent consensus statement of the American Society of Echocardiography. Detection of structural changes, such as Menkeber's sclerosis of the medial layer of the carotid artery, carotid aneurysm may indicate advanced vascular pathology and prompt an appropriate treatment. Ultrasound images of patients with atherosclerotic plaque and Menkeberg's sclerosis are presented and issues related to clinical significance of the common carotid artery intima-media thickness are discussed.
\end{abstract}

Key Words: B-mode carotid ultrasound, Atherosclerosis, Menkeberg's sclerosis, Inflammation.

\section{INTRODUCTION}

B-mode ultrasonography of the carotid artery is nowadays increasingly used as a non-invasive and wellvalidated tool for assessment of atherosclerotic burden in a variety of clinical conditions [1]. It is believed that it can be used for both research and clinical purposes.

Atherosclerotic changes in the carotid artery (e.g., thickened intima-media complex, presence of plaques, rough arterial wall, calcifications) and other structural abnormalities detected by high-resolution ultrasound have been shown to be strongly associated with a number of classic cardiovascular risk factors $[2,3]$. A prime example of this association is an increased prevalence of carotid plaques in patients with familial hypercholesterolemia and arterial hypertension (Fig. 1), warranting further investigations to assess the level of the carotid artery stenosis and tightening of lipidlowering, anti-hypertensive therapy and/or invasive surgical procedures. It should be emphasized that in the case of familial hypercholesterolemia visualization of substantial changes in carotid arteries such as atherosclerotic plaques may prompt the clinician to use ultrasound to search for other pathophysiologically related changes at other anatomical sites (e.g., Achilles tendon thickening) [4].

Evidence from a few well-designed, large scale prospective studies suggests that non-invasively measured intimamedia thickness (IMT) of the common carotid artery may predict occurrence of cardiovascular events in subjects without clinical manifestations of ischemic heart disease or cerebrovascular disease $[2,5]$ and in those with established cardiovascular disease [6]. Obviously, IMT values can be

*Address correspondence to this author at the Dudley Group of Hospitals NHS Trust, West Midlands DY1 2HQ, United Kingdom; Tel: +44-1384244842; Fax: +44-1384-244808;

E-mail: armen.gasparyan@dgoh.nhs.uk supplementary to cardiovascular risk assessments based on traditional risk factors and useful for cardiovascular drugs clinical efficiency assessments. Of note, in prospective studies with statins, carotid IMT proved to be essential for monitoring of the course of atherosclerotic vascular disease $[7,8]$.

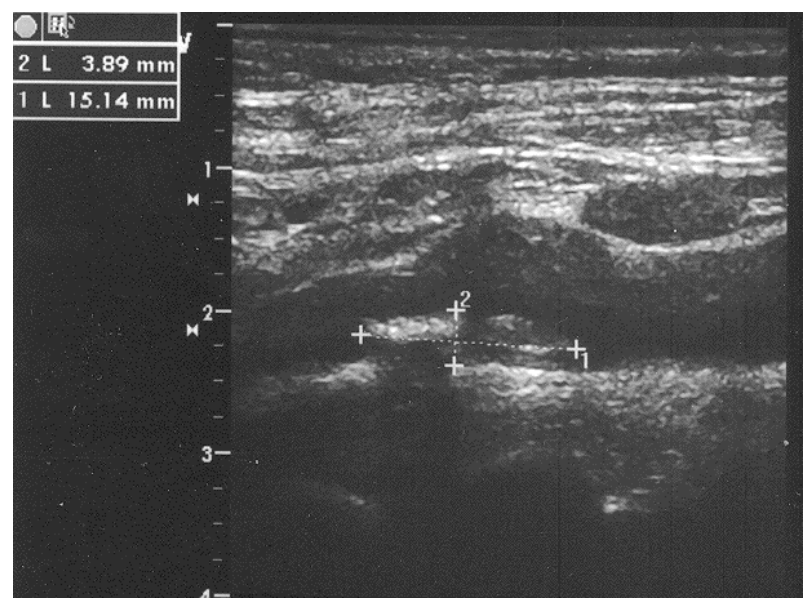

Fig. (1). Huge atherosclerotic plaque in the common carotid artery in a 77-yo Caucasian male patient with arterial hypertension, vertigo and carotid artery stenosis $(60 \%)$.

The latest consensus statement of the American Society of Echocardiography [9], based on the analysis of several prospective studies, confirmed that carotid IMT measured by high-resolution B-mode carotid ultrasound is a wellvalidated tool for detecting subclinical atherosclerosis and defining cardiovascular risk profile in conditions with low and high vascular risk. From practical standpoint, for the first time the statement firmly pointed out that the interpretation of IMT measurements should take into account age, sex, 
ethnicity, underlying clinical conditions (e.g., diabetes, peripheral vascular disease), duration and aggressiveness of cardiovascular drug treatments, notably with statins, and carotid scanning technique. The latter requires special professional skills and computer software, which can substantially improve accuracy of the lumen-intima interface detection and IMT measurement, and should be seen as a prerequisite of research studies. Accuracy of the measurement can be further improved by synchronized electrocardiography and $\mathrm{R}$ wave gating. Another practically important point in the statement was that far wall of the common carotid artery is the most appropriate site for IMT measurement, compared with other vascular sites. Calculation of IMT, however, should be mandatory accompanied by careful visualization of the whole system of extracranial carotid arteries to rule in or out presence of atherosclerotic plaques.

Outlined in the consensus statement the issue of age-related changes in the carotid arteries are of importance for further research studies, since it is well-known that predictive value of IMT and carotid plaques at a relatively young age (below 45) is still uncertain. However, more prospective studies are warranted to ascertain association between atherosclerotic changes of carotid arteries and hard points such as MI, stroke and death in different age groups.

It is also worth noting that there are uncertainties over the interpretation of IMT measurements and accuracy of cardiovascular risk calculation in diabetes mellitus and other lowor high-grade inflammatory disorders. The problem relates to the origin of structural changes in these clinical conditions. Atherosclerotic and inflammatory changes of the carotid artery are closely related and may alter the structure of intima-media complex in diverse directions. For example, in diabetes, disease-specific changes of the medial layer of the carotid artery, known as Menkeberg's sclerosis, may occur (Fig. 2), which further complicate interpretation of the value of IMT. Menkeberg's sclerosis reflects diabetic vascular pathology caused by long-standing underlying disease and, even if there is normal or slightly increased IMT, such as in the presented case, high risk of future cardiovascular events is obvious.

The impact of chronic inflammation on IMT of the carotid artery and the meaning of increased IMT in highgrade inflammatory diseases such as rheumatoid arthritis and systemic lupus erythematosus is another big issue, which, unfortunately, has not been covered in the statement.

It is believed that an increased IMT is a reflection of subclinical and accelerated atherosclerosis in chronic inflammatory conditions. However, it appears that links between inflammation and IMT are not straightforward. Although positive association of a variety of inflammatory biomarkers (first of all, $\mathrm{C}$ reactive protein and fibrinogen) with carotid IMT have been found in the vast majority of observational studies [10], there are also few negative publications [11], and it is still not clear whether an increase of IMT may independently predict cardiovascular events in high-grade inflammatory conditions [12]. Assessment of clinical efficiency and anti-atherosclerotic effects of widely used in these conditions anti-inflammatory and lipidlowering drugs (e.g., anti-TNFalpha drugs and statins) based on IMT values over the course of short- and long-term treatment is questionable. In this regard, a number of factors potentially influencing IMT values should be carefully weighed (e.g., duration and activity of an inflammatory disorder). A decrease of IMT over a short period of time (days-weeks) is most probably due to the dampening inflammation in the vascular wall rather than antiatherosclerotic effect of treatment. In fact, recently published results of the Measuring Effects on intima media Thickness: an Evaluation Of Rosuvastatin (METEOR) trial come to prove that the use of carotid ultrasound for assessment of anti-atherosclerotic effects of a drug treatment is justified when repetitive measurements over a long period of time (at least 12 months) are available [13].

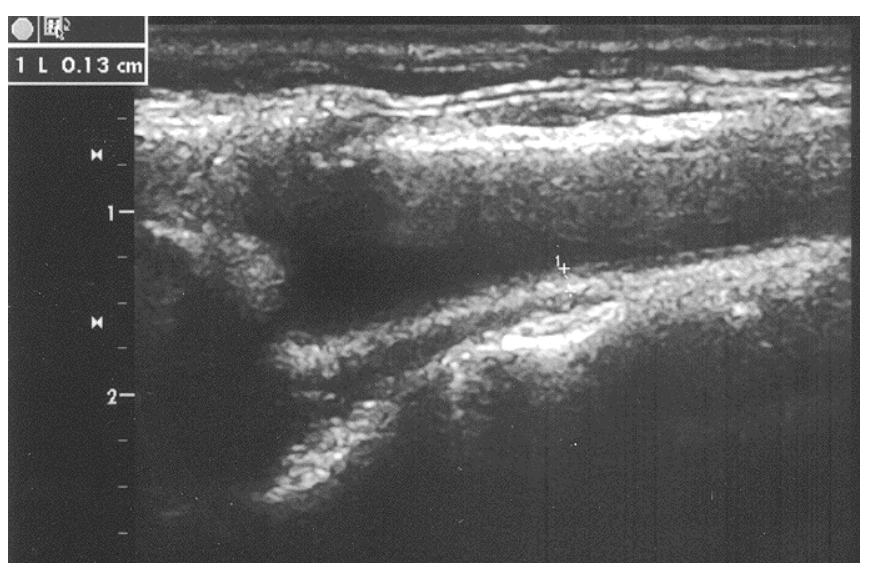

Fig. (2). Carotid ultrasonography of a 72-yo Caucasian female patient with a history of poorly contolled type 2 diabetes mellitus [duration - 38 years; fasting blood glucose - $283 \mathrm{mg} / \mathrm{dL}$ (reference range $-55-105 \mathrm{mg} / \mathrm{dL}$ ) and glycosilated hemoglobin $\mathrm{A}_{1 \mathrm{c}}-10.8 \%$ (3.4-6.1\%)]. IMT of the common carotid artery is $1.3 \mathrm{~mm}$; hyperechogeneity of the medial layer (suggestive of Menkeberg's medial sclerosis), soft plaques in bifurcation of the catorid artery; no signs of carotid stenosis.

Further long-term prospective studies are needed to address this issue in the context of high-grade inflammatory disorders and provide us with the clues over clinical significance of IMT changes in different clinical conditions. The latter may also lead to specific recommendations on the routine use of carotid ultrasound in clinical practice.

Carotid ultrasound assessments of atherosclerotic burden have been relatively well studied. Nevertheless, the use of this important tool may be further expanded towards detecting non-atherosclerotic changes as well. An appropriate example is carotid pathology in Behcet's disease, where visualization of the carotid aneurysm may indicate aggresive course of the disease and prompt further investigations to search for other sites of aneurysmatic vascular affections and proper treatment $[14,15]$.

\section{ACKNOWLEDGEMENTS}

The authors would like to thank Dr. Albert Holler and Dr. Eva Gebetsroither from the Department of Internal Medicine, Krankenhaus Barmherzigen Brüder, Graz Medical University for their valuable comments. Written informed consents were obtained from patients whose carotid ultrasound images were presented. 


\section{REFERENCES}

[1] Thompson JB, Blaha M, Resar JR, Blumenthal RS, Desai MY. Strategies to reverse atherosclerosis: an imaging perspective. Curr Treat Options Cardiovasc Med 2008; 10: 283-93.

[2] Li C, Engstrom G, Berglund G, Janzon L, Hedblad B. Incidence of ischemic stroke in relation to asymptomatic carotid artery atherosclerosis in subjects with normal blood pressure. A Prospective Cohort Study. Cerebrovasc Dis 2008; 26: 297-303.

[3] El-Gendi SS, Bakeet MY, El-Hamed EA, Ibrahim FK, Ahmed R. The value of lipoprotein (a), homocysteine, and Doppler of carotid and femoral arteries in assessment of atherosclerosis in asymptomatic cardiovascular risk patients. J Cardiol 2008; 52: 202-11.

[4] Kiortsis DN, Argyropoulou MI, Xydis V, Tsouli SG, Elisaf MS. Correlation of Achilles tendon thickness evaluated by ultrasonography with carotid intima-media thickness in patients with familial hypercholesterolemia. Atherosclerosis 2006; 186: 228-9.

[5] O'Leary DH, Polak JF, Kronmal RA, Manolio TA, Burke GL, Wolfson SK, Jr. Carotid-artery intima and media thickness as a risk factor for myocardial infarction and stroke in older adults. Cardiovascular Health Study Collaborative Research Group. N Engl J Med 1999; 340: 14-22.

[6] Zielinski T, Dzielinska Z, Januszewicz A, et al. Carotid intimamedia thickness as a marker of cardiovascular risk in hypertensive patients with coronary artery disease. Am J Hypertens 2007; 20: 1058-64.

[7] Kastelein JJ, de Groot E, Sankatsing R. Atherosclerosis measured by B-mode ultrasonography: effect of statin therapy on disease progression. Am J Med 2004; 116 (Suppl 6A): 31S-36S.

[8] Crouse JR, 3rd, Grobbee DE, O'Leary DH, et al. Measuring effects on intima media thickness: an evaluation of rosuvastatin in subclinical atherosclerosis--the rationale and methodology of the METEOR study. Cardiovasc Drugs Ther 2004; 18: 231-8.

[9] Stein JH, Korcarz CE, Hurst RT, et al. American Society of Echocardiography Carotid Intima-Media Thickness Task Force. Use of carotid ultrasound to identify subclinical vascular disease and evaluate cardiovascular disease risk: a consensus statement from the American Society of Echocardiography Carotid Intima-Media Thickness Task Force. Endorsed by the Society for Vascular Medicine. J Am Soc Echocardiogr 2008; 21: 93-111.

[10] Baldassarre D, De Jong A, Amato M, et al. Carotid intima-media thickness and markers of inflammation, endothelial damage and hemostasis. Ann Med 2008; 40: 21-44.

[11] Kiortsis DN, Tsouli S, Lourida ES, et al. Lack of association between carotid intima-media thickness and PAF-acetylhydrolase mass and activity in patients with primary hyperlipidemia. Angiology 2005; 56: 451-8.

[12] Veldhuijzen van Zanten JJ, Kitas GD. Inflammation, carotid intima-media thickness and atherosclerosis in rheumatoid arthritis. Arthritis Res Ther 2008; 10: 102.

[13] Crouse JR, 3rd, Raichlen JS, Riley WA, et al. METEOR Study Group. Effect of rosuvastatin on progression of carotid intimamedia thickness in low-risk individuals with subclinical atherosclerosis: the METEOR Trial. JAMA 2007; 297: 1344-53.

[14] Ohshima T, Miyachi S, Hattori K, et al. A case of giant common carotid artery aneurysm associated with vascular Behçet disease: successfully treated with a covered stent. Surg Neurol 2008; 69: 297-301.

[15] Alpagut U, Ugurlucan M, Dayioglu E. Major arterial involvement and review of Behcet's disease. Ann Vasc Surg 2007; 21: 232-9.

(c) Armen Yuri Gasparyan; Licensee Bentham Open.

This is an open access article licensed under the terms of the Creative Commons Attribution Non-Commercial License (http://creativecommons.org/licenses/by-nc/3.0/) which permits unrestricted, non-commercial use, distribution and reproduction in any medium, provided the work is properly cited. 